TITLE:

\title{
Measurement of kinetic energy release in CO fragmentation by charge-changing collisions of fast heavy ions
}

\section{$\operatorname{AUTHOR}(\mathrm{S}):$}

Mizuno, T.; Yamada, T.; Tsuchida, H.; Nakai, Y.; Itoh, A.

\section{CITATION:}

Mizuno, T.... [et al]. Measurement of kinetic energy release in CO fragmentation by chargechanging collisions of fast heavy ions. PHYSICAL REVIEW A 2010, 81(1): 012704.

\author{
ISSUE DATE: \\ 2010 \\ URL: \\ http://hdl.handle.net/2433/148387 \\ RIGHT: \\ (c) 2010 The American Physical Society
}




\title{
Measurement of kinetic energy release in $\mathrm{CO}$ fragmentation by charge-changing collisions of fast heavy ions
}

\author{
T. Mizuno, ${ }^{1}$ T. Yamada, ${ }^{1}$ H. Tsuchida, ${ }^{1,2}$ Y. Nakai, ${ }^{3}$ and A. Itoh ${ }^{1,2, *}$ \\ ${ }^{1}$ Department of Nuclear Engineering, Kyoto University, Kyoto 606-8501, Japan \\ ${ }^{2}$ Quantum Science and Engineering Center, Kyoto University, Kyoto 606-8501, Japan \\ ${ }^{3}$ RIKEN Nishina Center, Wako, Saitama 351-0198, Japan
}

(Received 25 May 2009; revised manuscript received 11 November 2009; published 21 January 2010)

\begin{abstract}
We study ionization and fragmentation of $\mathrm{CO}$ in electron loss and capture collisions of $\mathrm{B}^{2+}, \mathrm{O}^{2+}$, and $\mathrm{Si}^{2+}$ ions at an energy of $71.4 \mathrm{keV} / \mathrm{u}(v=1.69$ a.u. $)$. Coincidence measurements of fragment ions from $\mathrm{CO}$ and charge-selected ions were performed by means of a momentum three-dimensional imaging technique. Production cross sections of $\mathrm{CO}^{r+}$ and branching ratios into various fragmentation channels were obtained for $r=1-4$. We also measured kinetic energy release (KER) in individual fragmentation channels. The KER spectra for $r \leqslant 2$ are found to be different for electron loss and capture collisions, while the difference becomes small for $r \geqslant 3$. As a measure of the degree of molecular fragmentation, the magnitude of the binding energy of the relevant electronic states seems the important parameter both in loss and capture collisions.
\end{abstract}

DOI: 10.1103/PhysRevA.81.012704

PACS number(s): $34.50 . \mathrm{Gb}$

\section{INTRODUCTION}

In the last few decades collision-induced molecular fragmentation was extensively studied for various combinations between projectile particles and molecules [1-17]. The investigation was carried out for the identification of fragment-ion pairs, kinetic energies of the fragments, and the orientation effect on the molecular fragmentation. It is noted that experimental data of high quality can be obtained recently owing to the improvement of three-dimensional momentum imaging techniques $[18,19]$, where the momenta of individual fragment ions are measured three-dimensionally [3-8,11-15]. The molecular fragmentation occurs from dissociative excited states of an intact molecule that is mostly ionized to some degree in the case of ion impact. Thus, identification of fragment particles and their kinetic energies provide information about initial excited states of the molecular ion $[12,20]$. As the amount of excitation energy given to a target molecule depends on the impact parameter $(b)$ between collision partners, $b$-controlled experimental investigation is highly recommended for better understanding of the fragmentation process. However, such a kind of coincidence experiment is difficult particularly for fast heavy ions. Instead, the molecular fragmentation was investigated under conditions of charge-changing collisions by specifying the final projectile charge state [7-10,15-17] for both slow $(v<1$ a.u.) and fast $(v>1$ a.u.) ions.

In slow collisions of $\mathrm{He}^{2+}$ and $\mathrm{Ar}^{3+}$ with $\mathrm{N}_{2}$, Ehrich et al. [8] found that kinetic energy release (KER) spectra of two fragment $\mathrm{N}^{+}$ions are largely different when the final charge state is different. In collisions of slow $\mathrm{C}^{6+}$ and $\mathrm{Ar}^{11+}$ with CO [17], Wells et al. measured KER spectra and branching ratios of fragmentation channels. They showed that the branching ratios are almost constant over a wide range of projectile charge and velocity by using other available experimental data. On the other hand, the KER spectra are considerably different for different charge-changing collisions. It implies that different excited states of molecular ions are

\footnotetext{
*itoh@ nucleng.kyoto-u.ac.jp
}

populated transiently in accordance with their calculations of the extended over barrier model (ECOB). In electron capture collisions of slow highly charged ions with $\mathrm{D}_{2}$, Ali et al. [10] observed the three-body fragmentation effect between two $\mathrm{D}^{+}$ions and the projectile ion. In the case of high-energy collisions of $97 \mathrm{MeV} \mathrm{Ar}^{14+}+\mathrm{CO}$ [16], the average value of KER obtained in one-electron capture collisions was found to be considerably larger than that in pure ionization in which the incident charge state does not change. It suggests that the impact parameters relevant to pure ionization are larger than those to one-electron capture in fast highly charged ion impacts. In our recent study of $\mathrm{CO}$ fragmentation by $6 \mathrm{MeV} \mathrm{O}^{4+}$ ions [15], the mass spectra of fragment ions were found to change strongly according to both electron loss and capture collisions and was also affected largely by molecular orientation.

To achieve a more precise understanding of the difference between loss and capture collisions the investigation of $\mathrm{CO}$ fragmentation is extended to three incident ions of $\mathrm{B}^{2+}$, $\mathrm{O}^{2+}$, and $\mathrm{Si}^{2+}$ at the same impact energy of $71.4 \mathrm{keV} / \mathrm{u}$ $(v=1.69$ a.u.). Since the ionization potentials of these ions are different, the projectile ionization (electron loss) is expected to occur at different impact parameters leading to different features of the molecular fragmentation. Measurements were performed by using a three-dimensional momentum-imaging technique for both single-electron capture (1e capture) and single-electron loss ( $1 e$ loss) collisions. We present here the production cross sections of $\mathrm{CO}^{r+}(r=1-4)$ and branching ratios into various fragmentation channels. The KER spectra are also presented for individual fragmentation channels.

\section{EXPERIMENT}

Figure 1 shows a schematic diagram of the experimental apparatus installed at the Quantum Science and Engineering Center (QSEC) 1.7 MV tandem accelerator facility of Kyoto University $[15,21]$. Incident particles investigated here are $771 \mathrm{keV} \mathrm{B}^{2+}, 1.14 \mathrm{MeV} \mathrm{O}^{2+}$, and $2.0 \mathrm{MeV} \mathrm{Si}^{2+}$. All these ions have the same velocity of $v=1.69$ a.u. A beam from the 


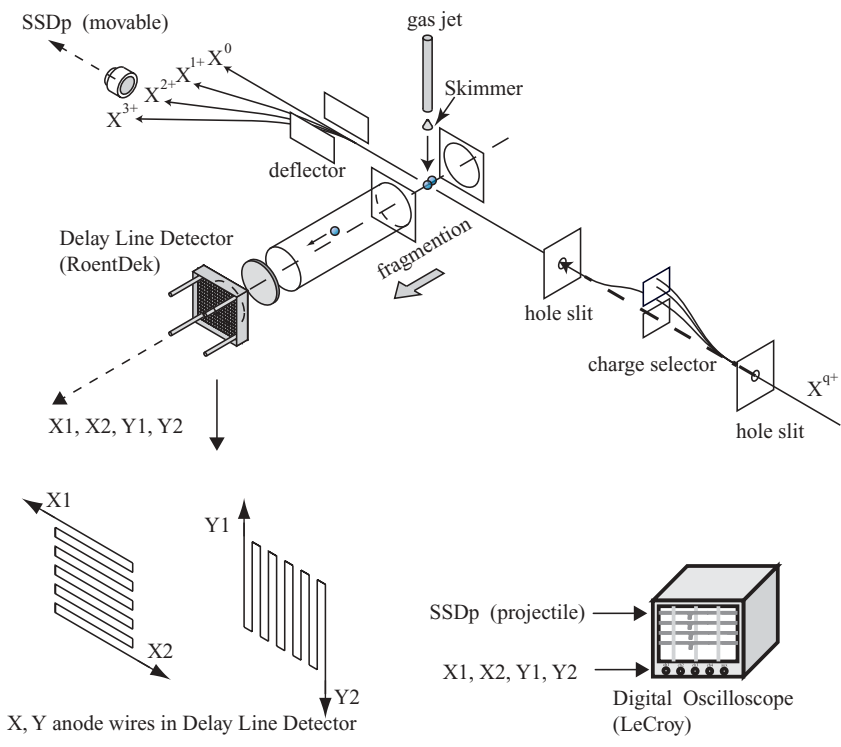

FIG. 1. (Color online) Experimental setup.

accelerator was carefully collimated to smaller than $1 \mathrm{~mm}$ in diameter and was crossed with an effusive molecular beam target of carbon monoxide. A base pressure of the collision chamber was about $1 \times 10^{-5} \mathrm{~Pa}$. Final projectile charge states were separated by an electrostatic deflector and detected by a movable solid state detector (SSD). In coincidence with these charge-selected particles the mass spectra of fragment ions from $\mathrm{CO}$ were obtained by a time of flight (TOF) method under a Wiley-McLaren spatial focusing condition. Fragment ions were extracted by an electric field of $423 \mathrm{~V} / \mathrm{cm}$ and detected by a two-stage multichannel plate (MCP) in conjunction with a position and time-sensitive delay-line anode. This extraction electric field was high enough to collect all the product ions emitted over all angles of $4 \pi$ sr solid angles. Four Mo grid meshes were used in the TOF spectrometer. The transmission rate per grid was 0.885 , giving rise to $(0.885)^{4}=0.613$ as the total transmission rate. The front plate of the MCP was biased to a negative voltage of $-2.4 \mathrm{kV}$, which is high enough to detect all the fragment ions investigated here [22]. The open ratio of the MCP was 0.57 . The event-by-event recording was achieved with a digital storage oscilloscope (WavePro7000, LeCroy). A typical example of TOF two-dimensional maps is shown in Fig. 2, exhibiting correlations of fragment-ion pairs produced simultaneously. Fragment ions arising from various ion pairs such as $\left(\mathrm{C}^{+}, \mathrm{O}^{+}\right),\left(\mathrm{C}^{2+}, \mathrm{O}^{+}\right),\left(\mathrm{C}^{+}, \mathrm{O}^{2+}\right)$, and $\left(\mathrm{C}^{2+}, \mathrm{O}^{2+}\right)$ are well separated from one another. From a series of such two-dimensional maps we obtained relative production cross sections $\sigma_{r}$ of $\mathrm{CO}^{r+}(r=1-4)$ and their branching ratios into individual fragmentation channels. Careful attention was paid to the detection efficiency as described in the following.

First, the detection efficiency $D$ for a single ion is given by the product of the grid transmission rate multiplied by the open ratio of the MCP, as $D=0.613 \times 0.57=0.35$. Hence, in the case of two-ion detection such as $\mathrm{CO}^{2+*} \rightarrow \mathrm{C}^{+}+\mathrm{O}^{+}$, the detection efficiency is $D^{2}$ and the cross section for this fragmentation channel is

$$
\sigma\left(\mathrm{C}^{+}, \mathrm{O}^{+}\right)=Y\left(\mathrm{C}^{+}, \mathrm{O}^{+}\right) / D^{2},
$$

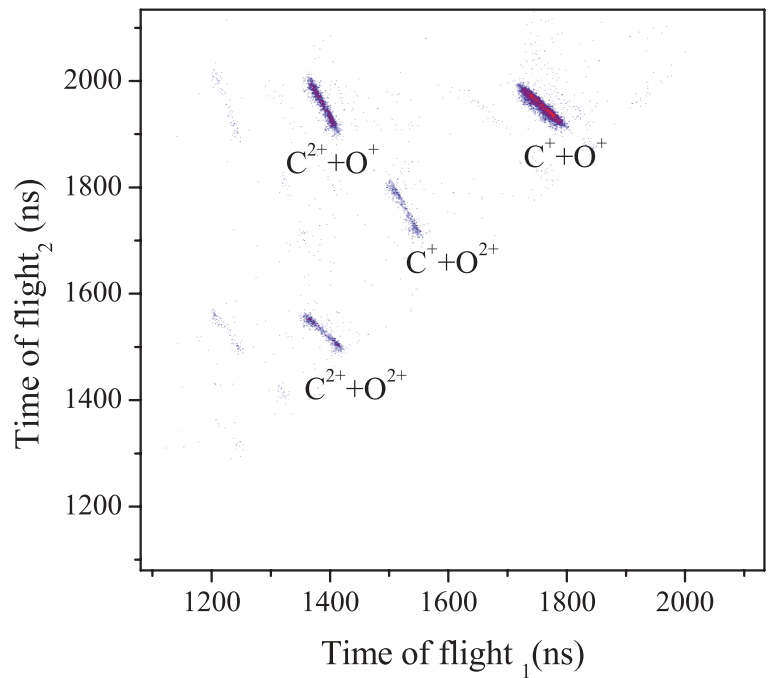

FIG. 2. (Color online) Two-dimensional TOF spectra obtained for $1 e$ loss collisions of $2 \mathrm{MeV} \mathrm{Si}{ }^{2+}$ with $\mathrm{CO}$.

with $Y$ the yield of two-ion detection in coincidence. As for the single-ion detection such as $\mathrm{CO}^{+*} \rightarrow \mathrm{C}^{+}+\mathrm{O}$, the correction is somewhat cumbersome because the yield $Y\left(\mathrm{C}^{+}\right)$contains contributions from other single-ion detection arising from fragmentation channels of $\left(\mathrm{C}^{+}, \mathrm{O}^{+}\right)$and $\left(\mathrm{C}^{+}, \mathrm{O}^{2+}\right)$ in which the $\mathrm{O}$ ion escapes from detection. In this case, the relationship of the yields is written as

$$
Y\left(\mathrm{C}^{+}\right)=\sigma\left(\mathrm{C}^{+}, \mathrm{O}\right) D+\left[\sigma\left(\mathrm{C}^{+}, \mathrm{O}^{+}\right)+\sigma\left(\mathrm{C}^{+}, \mathrm{O}^{2+}\right)\right] D(1-D) .
$$

The cross sections in the right-hand side are obtained individually, similar to Eq. (1). In this way, the partial cross sections or the branching ratios were deduced from experimental coincidence yields. Note that we ignored contributions from four-fold ionization events such as $\left(\mathrm{C}^{+}, \mathrm{O}^{3+}\right)$ because these events were smaller than $1 \%$ at most.

We also obtained KER of individual fragment ions from positions $(x, y)$ and flight time $t$ of these products. The energy resolution of KER was about $1 \mathrm{eV}$.

\section{RESULTS AND DISCUSSION}

TOF spectra of fragment ions produced in electron capture and loss collisions are shown in Fig. 3, illustrating different features of the molecular fragmentation according to the projectile species and the type of charge-changing collisions. First, a remarkable feature seen in the spectra is that the molecular fragmentation is enhanced strongly in $1 e$ loss collisions compared with $1 e$ capture collisions for all the incident ions. Second, the fragmentation is induced most strongly by $\mathrm{O}^{2+}$ impact as seen typically in the spectra of $1 e$ loss collisions. These characteristic features are understood more explicitly from production cross sections $\sigma_{r}$ and their branching ratios given in Table I. Here, the cross sections are relative values with $\Sigma_{r=1}^{4} \sigma_{r}=100$ for each charge-changing collision. It is found that the survival probabilities of intact parent ions $\mathrm{CO}^{r+}$ decrease rapidly with increasing $r$ for all the 
TABLE I. Relative production cross sections $\sigma_{r}$ of $\mathrm{CO}^{r+}(r=1-4)$ and branching ratios into various fragmentation channels measured for $1 e$ capture and $1 e$ loss collisions of $\mathrm{Si}^{2+}, \mathrm{B}^{2+}$, and $\mathrm{O}^{2+}$ ions at $v=1.69$ a.u. $(71.4 \mathrm{keV} / \mathrm{u})$. All values are given in $\%$. Experimental data obtained by other groups $[1,11]$ are also listed for comparison.

\begin{tabular}{|c|c|c|c|c|c|c|c|c|}
\hline & \multicolumn{2}{|c|}{$\mathrm{Si}^{2+}$} & \multicolumn{2}{|c|}{$\mathrm{B}^{2+}$} & \multicolumn{2}{|c|}{$\mathrm{O}^{2+}$} & \multirow[t]{2}{*}{$865 \mathrm{MeV} \mathrm{Xe} e^{44+}[11]$} & \multirow[t]{2}{*}{$19 \mathrm{MeV} \mathrm{F}^{4+}[1]$} \\
\hline & $1 e$ cap & $1 e$ loss & $1 e$ cap & $1 e$ loss & $1 e$ cap & $1 e$ loss & & \\
\hline$r=1 \sigma_{1}$ & $75 \pm 0.4$ & $48 \pm 0.4$ & $56 \pm 0.4$ & $54 \pm 0.3$ & $66 \pm 0.3$ & $34 \pm 0.4$ & $75.3 \pm 5.5$ & $78.6 \pm 0.2$ \\
\hline $\mathrm{CO}^{+}$ & $76 \pm 0.4$ & $42 \pm 0.4$ & $65 \pm 0.4$ & $46 \pm 0.3$ & $47 \pm 0.4$ & $25 \pm 0.4$ & $73.1 \pm 2.4$ & $77.0 \pm 0.2$ \\
\hline $\mathrm{C}^{+}+\mathrm{O}^{0}$ & $14 \pm 0.03$ & $30 \pm 0.2$ & $18 \pm 0.1$ & $30 \pm 0.1$ & $26 \pm 0.1$ & $39 \pm 0.3$ & $15 \pm 1.2$ & $12.2 \pm 1.2$ \\
\hline $\mathrm{C}+\mathrm{O}^{+}$ & $10 \pm 0.1$ & $28 \pm 0.2$ & $17 \pm 0.2$ & $24 \pm 0.2$ & $27 \pm 0.8$ & $36 \pm 0.4$ & $12 \pm 1.8$ & $10.8 \pm 1.0$ \\
\hline$r=2 \sigma_{2}$ & $22 \pm 0.4$ & $36 \pm 0.5$ & $33 \pm 0.4$ & $33 \pm 0.4$ & $22 \pm 0.5$ & $41 \pm 0.6$ & $13.1 \pm 2.1$ & $14.4 \pm 1.7$ \\
\hline $\mathrm{CO}^{2+}$ & $12 \pm 0.3$ & $5 \pm 0.1$ & $10 \pm 0.2$ & $7 \pm 0.2$ & $16 \pm 0.4$ & $5 \pm 0.2$ & $5.3 \pm 1.0$ & $6.0 \pm 0.6$ \\
\hline $\mathrm{C}^{2+}+\mathrm{O}^{0}$ & $8 \pm 0.1$ & $18 \pm 0.2$ & $13 \pm 0.1$ & $13 \pm 0.2$ & $24 \pm 0.3$ & $24 \pm 0.2$ & $11.2 \pm 6.9$ & $13.8 \pm 2.0$ \\
\hline $\mathrm{C}^{0}+\mathrm{O}^{2+}$ & $4 \pm 0.1$ & $11 \pm 0.2$ & $8 \pm 0.1$ & $10 \pm 0.1$ & $15 \pm 0.2$ & $19 \pm 0.2$ & $1.8 \pm 4.1$ & $6.6 \pm 1.2$ \\
\hline $\mathrm{C}^{+}+\mathrm{O}^{+}$ & $76 \pm 1.2$ & $66 \pm 0.9$ & $69 \pm 0.9$ & $70 \pm 0.9$ & $45 \pm 1.1$ & $53 \pm 0.9$ & $81.7 \pm 6.2$ & $73.6 \pm 8.0$ \\
\hline$r=3 \sigma_{3}$ & $3 \pm 0.1$ & $13 \pm 0.3$ & $9 \pm 0.3$ & $10 \pm 0.3$ & $9 \pm 0.4$ & $17 \pm 0.5$ & $5.8 \pm 1.0$ & $4.9 \pm 0.6$ \\
\hline $\mathrm{C}^{2+}+\mathrm{O}^{+}$ & $82 \pm 3.4$ & $68 \pm 1.5$ & $75 \pm 1.8$ & $73 \pm 1.6$ & $75 \pm 2.2$ & $71 \pm 1.6$ & $63.2 \pm 8.8$ & $66.6 \pm 7.0$ \\
\hline $\mathrm{C}^{+}+\mathrm{O}^{2+}$ & $18 \pm 1.6$ & $32 \pm 1.0$ & $25 \pm 1.0$ & $27 \pm 1.0$ & $25 \pm 1.2$ & $29 \pm 1.0$ & $29.7 \pm 3.0$ & $27.3 \pm 3.0$ \\
\hline $\mathrm{C}^{3+}+\mathrm{O}^{0}$ & - & - & - & - & - & - & $7.1 \pm 5.2$ & $5.9 \pm 1.0$ \\
\hline $\mathrm{C}^{0}+\mathrm{O}^{3+}$ & - & - & - & - & - & - & - & $0.2 \pm 0.5$ \\
\hline$r=4 \sigma_{4}$ & - & $3 \pm 0.1$ & $2 \pm 0.1$ & $3 \pm 0.1$ & $3 \pm 0.1$ & $8 \pm 0.2$ & $2.9 \pm 0.3$ & $2.1 \pm 0.3$ \\
\hline $\mathrm{C}^{2+}+\mathrm{O}^{2+}$ & - & 100 & 100 & 100 & 100 & 100 & $81 \pm 7.5$ & $75.0 \pm 8.0$ \\
\hline $\mathrm{C}^{+}+\mathrm{O}^{3+}$ & - & - & - & - & - & - & $4 \pm 1.3$ & $6.2 \pm 0.7$ \\
\hline $\mathrm{C}^{3+}+\mathrm{O}^{+}$ & - & - & - & - & - & - & $15 \pm 2.3$ & $17.0 \pm 1.8$ \\
\hline
\end{tabular}

incident ions. As for $r=3$, all the parent ions cannot remain intact but decay to fragments.

Qualitatively to say, these different features of the molecular fragmentation may be attributed to the different amounts of inelastic energy transferred to the target particle. For instance, ionization of a projectile electron (electron loss) by a neutral molecule is supposed to occur in smaller impact-parameter collisions than electron-capture collisions [6]. This is because a certain amount of inelastic energy is required to remove a tightly bound projectile electron. Consequently, it leads to a larger amount of energy transfer in loss collisions and more violent molecular fragmentation is expected to occur. Note that the binding energies of the outermost electrons of $\mathrm{Si}^{2+}, \mathrm{B}^{2+}$, and $\mathrm{O}^{2+}$ are $33.5,37.9$, and $54.9 \mathrm{eV}$, respectively. It is interesting to point out that the degree of molecular fragmentation in $1 e$ loss collisions reflects fairly well the

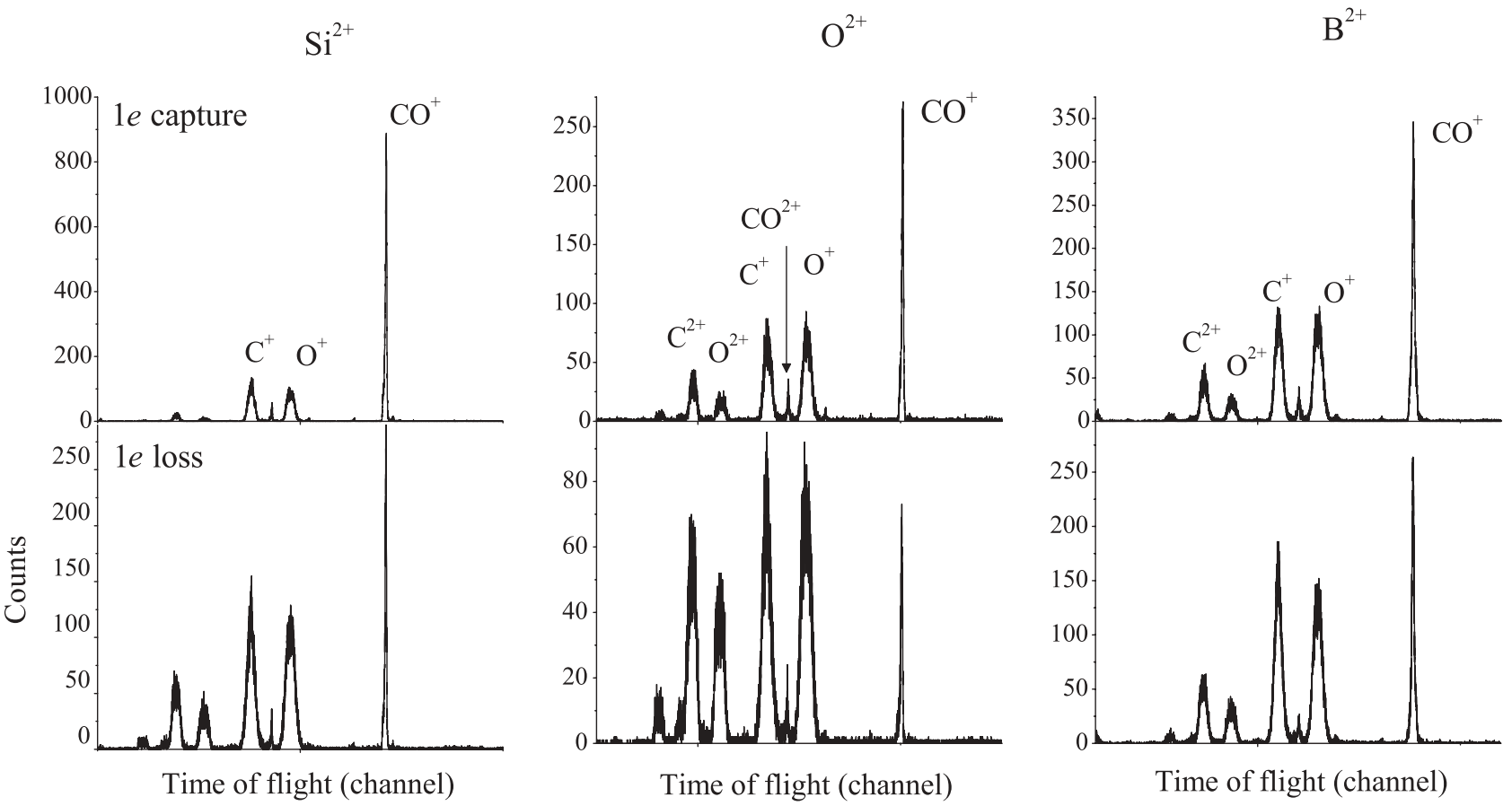

FIG. 3. TOF spectra of fragment ions from $\mathrm{CO}$ produced in $1 e$ capture (upper) and $1 e$ loss (lower) collisions of $\mathrm{Si}^{2+}, \mathrm{O}^{2+}$, and $\mathrm{B}^{2+}$ ions. 
magnitude of these binding energies. It implies that the effective impact parameters in $\mathrm{O}^{2+}$ collisions are smaller than other projectiles because of the larger binding energy of $\mathrm{O}^{2+}$. Nearly the same spectral profile and the branching ratios observed for the impact of $\mathrm{Si}^{2+}$ and $\mathrm{B}^{2+}$ are reasonably attributed to equivalent binding energies of these ions.

In electron-capture collisions, similar spectral profiles and branching ratios were obtained for $\mathrm{O}^{2+}$ and $\mathrm{B}^{2+}$ impacts, while the molecular fragmentation as well as multiple ionization are considerably suppressed in $\mathrm{Si}^{2+}$ impact. We interpret this as in the following. If we assume that the projectile ion captures an electron into its ground state, the capture states are $2 s$ in $\mathrm{B}^{+}\left(1 s^{2} 2 s^{2}\right), 2 p$ in $\mathrm{O}^{+}\left(1 s^{2} 2 s^{2} 2 p^{3}\right)$ and $3 p$ in $\mathrm{Si}^{+}\left([\mathrm{Ne}] 3 s^{2} 3 p^{1}\right)$, respectively. The binding energies of these states are $25.2 \mathrm{eV}$ $\left(\mathrm{B}^{+}\right), 31.1 \mathrm{eV}\left(\mathrm{O}^{+}\right)$, and $16.3 \mathrm{eV}\left(\mathrm{Si}^{+}\right)$. As the principal quantum number of the capture states are $n=2$ for $\mathrm{B}^{2+}$ and $\mathrm{O}^{2+}$ and $n=3$ for $\mathrm{Si}^{2+}$ ions, electron capture by $\mathrm{Si}^{2+}$ is supposed to take place at a larger distance resulting in less fragmentation and less multiple ionization. It is interesting to point out that the degree of the molecular fragmentation also reflects the magnitude of the binding energy of the capture states.

As for $\mathrm{CO}^{3+}$, fragmentation into the channel $\left(\mathrm{C}^{2+}, \mathrm{O}^{+}\right)$ is significantly larger than the channel $\left(\mathrm{C}^{+}, \mathrm{O}^{2+}\right)$ for all the incident ions. This can be attributed to the smaller total ionization energy required to produce these states due to the smaller ionization potentials of the $\mathrm{C}$ compared to the $\mathrm{O}$ atom. Note that a simple sum of the ionization potentials required for these ionized states shows 49.3 and $59.9 \mathrm{eV}$ for $\left(\mathrm{C}^{2+}, \mathrm{O}^{+}\right)$ and $\left(\mathrm{C}^{+}, \mathrm{O}^{2+}\right)$, respectively.

Table I also presents other experimental TOF data obtained by pulsed-beam coincidence methods in which individual charge-changing processes are not distinguished [1,11]. Their data of both production cross sections and branching ratios are in good agreement with each other irrespective of largely different incident species, velocities, and charges. It is interesting to note that our charge-changing data are greatly different from their results. Despite that, the $1 e$ capture data by $\mathrm{Si}^{2+}$ impact seem close to their results, indicating that the $1 e$ capture may be the dominant process in their collision systems as well.

Figure 4 compares KER spectra in $\mathrm{CO}^{2+*} \rightarrow \mathrm{C}^{+}+\mathrm{O}^{+}$, exhibiting considerable differences between $1 e$ loss and capture collisions. The peak energy and broadness of the spectra are both larger in $1 e$ loss than $1 e$ capture collisions, implying the population of higher excited states of $\mathrm{CO}^{r+*}$ in loss collisions. This trend is also found for $\mathrm{N}_{2}$ fragmentation in charge-changing collisions of $300 \mathrm{keV} \mathrm{Ar}^{3+}$ ions [8]. In collisions of $97 \mathrm{MeV} \mathrm{Ar}^{14+}$ with $\mathrm{CO}$, Watson et al. reported that the average values of KER in $1 e$ capture is considerably larger than direct ionization [16]. Also, multiple ionization and the degree of fragmentation are both enhanced largely in $1 e$ capture collisions. These results imply obviously that, in fast collisions ( $v=9.8$ a.u.), the effective impact parameter is small in electron capture compared to direct ionization.

Figure 5 shows KER spectra in various fragmentation channels from $\mathrm{CO}^{r+*}$ produced by $2 \mathrm{MeV} \mathrm{Si}^{2+}$, where we also show two-electron loss data. The solid square curves are theoretical spectra estimated from a simple Coulomb explosion model using $E_{c}=q_{1} q_{2} / R_{e}$, where $E_{c}$ is the central peak energy, $q$ the charges of ion pairs, and $R_{e}$ the equilibrium

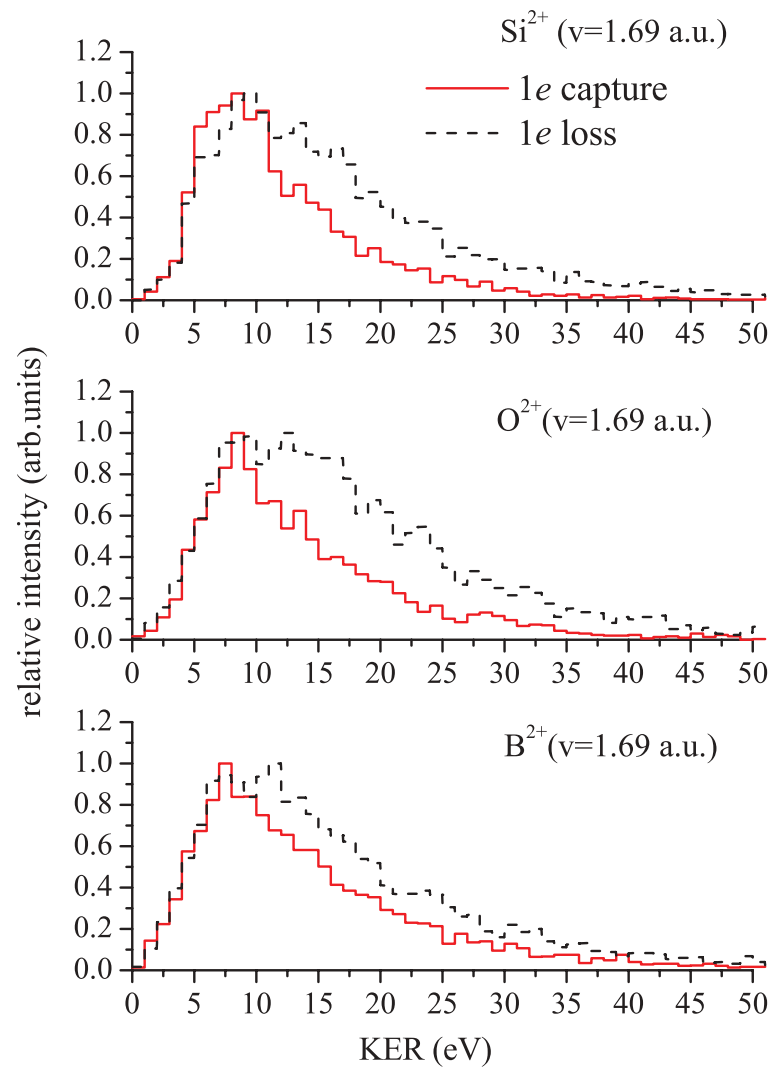

FIG. 4. (Color online) Comparison of KER spectra in $\mathrm{CO}^{2+} \rightarrow \mathrm{C}^{+}+\mathrm{O}^{+}$obtained in charge-changing collisions of $\mathrm{Si}^{2+}$, $\mathrm{O}^{2+}$, and $\mathrm{B}^{2+}$ ions.

internuclear distance of CO. The width $W$ of the distribution is related to the probability density of the ground state of $\mathrm{CO}$ and may be estimated to the first approximation, as [4]

$$
W \simeq-\frac{d E}{d R} \delta R=\frac{q_{1} q_{2}}{R_{e}^{2}} \delta R_{e},
$$

TABLE II. Theoretical KER values predicted from potential energy curves $[2,14,20]$.

\begin{tabular}{lcc}
\hline \hline Electronic state & KER $(\mathrm{eV})$ & Channel \\
\hline${ }^{1} \Pi$ & 5.8 & $\mathrm{C}^{+}\left({ }^{2} P\right)-\mathrm{O}^{+}\left({ }^{4} S\right)$ \\
${ }^{3} \Sigma^{+}$ & 7.8 & $\mathrm{C}^{+}\left({ }^{2} P\right)-\mathrm{O}^{+}\left({ }^{4} S\right)$ \\
$2^{1} \Sigma^{+}$ & 9.5 & $\mathrm{C}^{+}\left({ }^{2} P\right)-\mathrm{O}^{+}\left({ }^{4} S\right)$ \\
${ }^{2} \Pi$ & 16.1 & $\mathrm{C}^{2+}\left({ }^{3} P\right)-\mathrm{O}^{+}\left({ }^{4} S\right)$ \\
${ }^{4} \Pi$ & 17.9 & $\mathrm{C}^{2+}\left({ }^{3} P\right)-\mathrm{O}^{+}\left({ }^{4} S\right)$ \\
${ }^{6} \Pi$ & 25.9 & $\mathrm{C}^{2+}\left({ }^{3} P\right)-\mathrm{O}^{+}\left({ }^{4} S\right)$ \\
${ }^{2} \Sigma^{+}$ & 20.3 & $\mathrm{C}^{2+}\left({ }^{1} S\right)-\mathrm{O}^{+}\left({ }^{2} D\right)$ \\
${ }^{2} \Sigma^{+}$ & 17.5 & $\mathrm{C}^{2+}\left({ }^{3} P\right)-\mathrm{O}^{+}\left({ }^{4} S\right)$ \\
${ }^{2} \Sigma^{-}$ & 19.6 & $\mathrm{C}^{2+}\left({ }^{1} S\right)-\mathrm{O}^{+}\left({ }^{2} D\right)$ \\
${ }^{2} \Sigma^{-}$ & 16.8 & $\mathrm{C}^{2+}\left({ }^{3} P\right)-\mathrm{O}^{+}\left({ }^{4} S\right)$ \\
${ }^{4} \Sigma^{-}$ & 22.7 & $\mathrm{C}^{2+}\left({ }^{1} S\right)-\mathrm{O}^{+}\left({ }^{4} S\right)$ \\
${ }^{4} \Sigma^{-}$ & 16.2 & $\mathrm{C}^{2+}\left({ }^{3} P\right)-\mathrm{O}^{+}\left({ }^{4} S\right)$ \\
${ }^{2} \Delta$ & 21.3 & $\mathrm{C}^{2+}\left({ }^{1} S\right)-\mathrm{O}^{+}\left({ }^{2} D\right)$ \\
${ }^{3} \Pi$ & 41.1 & $\mathrm{C}^{2+}\left({ }^{1} S\right)-\mathrm{O}^{2+}\left({ }^{3} P\right)$ \\
${ }^{3} \Delta$ & 45.4 & $\mathrm{C}^{2+}\left({ }^{1} S\right)-\mathrm{O}^{2+}\left({ }^{3} P\right)$ \\
${ }^{1} \Sigma$ & 45.3 & $\mathrm{C}^{2+}\left({ }^{3} P\right)-\mathrm{O}^{2+}\left({ }^{3} P\right)$ \\
${ }^{1} \Delta$ & 47.0 & $\mathrm{C}^{2+}\left({ }^{3} P\right)-\mathrm{O}^{2+}\left({ }^{3} P\right)$ \\
\hline \hline
\end{tabular}



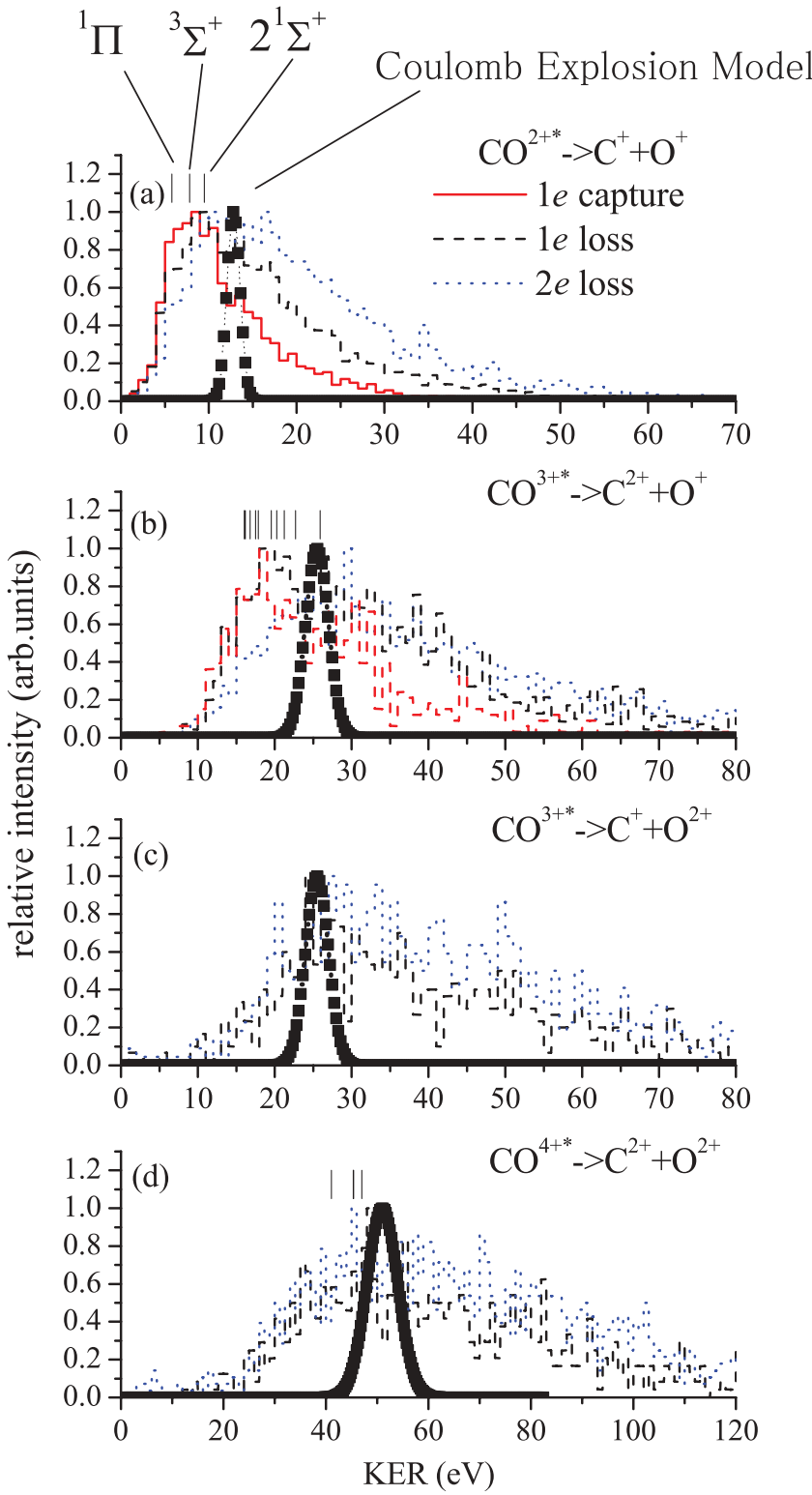

FIG. 5. (Color online) KER spectra in the fragmentation of $\mathrm{CO}^{r+}$ $(r=2,3,4)$ into various channels by the $2.0 \mathrm{MeV} \mathrm{Si}^{2+}$ impact. Full square curves are calculations with a pure Coulomb explosion model and vertical lines indicate predicted values from accurate potential surfaces $[2,14,20]$. where we use $R_{e} \simeq 2.1$ and $\delta R_{e}=0.18$ a.u. [11]. It is seen that the central energy $E_{c}$ is reasonably close to the peak position of each experimental spectrum. However, the width of the experimental distributions is completely larger than the estimation. Inadequacy of this simple Coulomb repulsion model is well known as reported in previous work $[4,11,12,14]$. It should be pointed out that this model does not hold even for the highest charge state $\mathrm{CO}^{4+*}$.

Theoretical KER predicted from potential energy curves of possible electronic states $[2,14,20]$ are listed in Table II and partly shown by vertical lines in Fig. 5. Excited states of $\mathrm{CO}^{2+*}$ are ${ }^{1} \Pi,{ }^{3} \Sigma$, and $2^{1} \Sigma^{+}$and are consistent with other experimental results obtained for $11.4 \mathrm{MeV} / \mathrm{u} \mathrm{O}^{7+}$ [12]. As for the fragmentation of $\mathrm{CO}^{3+}$, the fragmentation channel $\left(\mathrm{C}^{+}, \mathrm{O}^{2+}\right)$ shown in panel (c) are somewhat broader to higher KER compared with $\left(\mathrm{C}^{2+}, \mathrm{O}^{+}\right)$shown in panel (b). This is reasonable because the adiabatic potential energy of $\left(\mathrm{C}^{+}, \mathrm{O}^{2+}\right)$ is known to be higher than the latter channel at $R \rightarrow \infty$ [2]. This trend is also observed in other experimental results [11,14]. In the case of $\mathrm{CO}^{4+}$ fragmentation into $\left(\mathrm{C}^{2+}, \mathrm{O}^{2+}\right)$ shown in panel (d), the KER spectra are essentially the same for both $1 e$ loss and $2 \mathrm{e}$ loss collisions, implying an equivalent excitation of parent ions in these collisions.

In summary, we investigated the fragmentation of $\mathrm{CO}$ in charge-changing collisions of $\mathrm{B}^{2+}, \mathrm{O}^{2+}$, and $\mathrm{Si}^{2+}$ ions with the same incident velocity of $v=1.69$ a.u. It is found that the degree of molecular fragmentation in electron loss collisions becomes large as the binding energy of the projectile outermost electron increases. Similarly, in electron capture collisions the degree of fragmentation increases as the binding energy of the capture state increases. This implies that the effective impact parameters are different in these collisions, leading to a different energy transfer to a $\mathrm{CO}$ molecule. The KER spectra in the fragmentation of $\mathrm{CO}^{r+*}$ are found to be different for electron loss and capture collisions, while the difference becomes small for $r \geqslant 3$.

\section{ACKNOWLEDGMENTS}

This work was supported by Grant-in-Aid for Scientific Research (B) No. 19360427 from the Japan Society for the Promotion of Science.
[1] I. Ben Itzhak, S. G. Ginther, and K. D. Carnes, Phys. Rev. A 47, 2827 (1993).

[2] D. Mathur, E. Krishnakumar, K. Nagesha, V. R. Marathe, V. Krishnamurthi, F. A. Rajgara, and U. T. Raheja, J. Phys. B: At. Mol. Opt. Phys. 26, L141 (1993).

[3] U. Werner, N. M. Kabachnik, V. N. Kondratyev, and H. O. Lutz, Phys. Rev. Lett. 79, 1662 (1997).

[4] B. Siegmann, U. Werner, R. Mann, N. M. Kabachnik, and H. O. Lutz, Phys. Rev. A 62, 022718 (2000).

[5] B. Siegmann, U. Werner, H. Lebius, B. Huber, H. O. Lutz, and R. Mann, Nucl. Instrum. Methods B 205, 629 (2003).
[6] S. De, P. N. Ghosh, A. Roy, and C. P. Safvan, Nucl. Instrum. Methods B 243, 435 (2006).

[7] I. Ali et al., Nucl. Instrum. Methods B 149, 490 (1999).

[8] M. Ehrich, B. Siegmann, U. Werner, and H. Lebius, Rad. Phys. Chem. 68, 127 (2003).

[9] T. Kaneyasu, T. Azuma, and K. Okuno, J. Phys. B 38, 1341 (2005).

[10] I. Ali, R. D. DuBois, C. L. Cocke, S. Hagmann, C. R. Feeler, and R. E. Olson, Phys. Rev. A 64, 022712 (2001).

[11] L. Adoui, C. Caraby, A. Cassimi, D. Lelièvre, J. P. Grandin, and A. Dubois, J. Phys. B 32, 631 (1999). 
[12] M. Tarisien et al., J. Phys. B 33, L11 (2000).

[13] B. Siegmann, U. Werner, Z. Kaliman, Z. Roller-Lutz, N. M. Kabachnik, and H. O. Lutz, Phys. Rev. A 66, 052701 (2002).

[14] J. Rajput and C. P. Safvan, Phys. Rev. A 75, 062709 (2007).

[15] T. Mizuno, T. Majima, H. Tsuchida, Y. Nakai, and A. Itoh, J. Phys.: Conf. Ser. 58, 173 (2007).

[16] R. L. Watson, G. Sampoll, V. Horvat, and O. Heber, Phys. Rev. A 53, 1187 (1996).

[17] E. Wells, T. Nishide, H. Tawara, R. L. Watson, K. D. Carnes, and I. Ben Itzhak, Phys. Rev. A 77, 064701 (2008).
[18] R. Döner et al., Phys. Rep. 330, 95 (2000).

[19] J. Ullrich, R. Moshammer, A. Dorn, R. Döner. Schmidt, and H. Schmidt-Böcking, Rep. Prog. Phys. 66, 1463 (2003).

[20] M. Lundqvist, P. Baltzer, D. Edvardsson, L. Karlsson, and B. Wannberg, Phys. Rev. Lett. 75, 1058 (1995).

[21] T. Mizuno, T. Yamada, H. Tsuchida, Y. Nakai, and A. Itoh, J. Phys.: Conf. Ser. 163, 012039 (2009).

[22] A. Itoh, H. Tsuchida, T. Majima, and N. Imanishi, Phys. Rev. A 59, 4428 (1999). 